\title{
Editorial
}

https://doi.org/10.25100/iyc.v19i2.5923

\section{Futuro de las publicaciones científicas en Colombia}

Como es del conocimiento de todos los interesados, Publindex a mediados de 2016 dio apertura a la etapa II "Clasificación oficial" de la convocatoria para la evaluación de revistas científicas de acuerdo con la construcción conceptual definida en los documentos: "Política para mejorar la calidad de las publicaciones científicas y en el Modelo de clasificación de revistas científicas - 2016”.

Los resultados de la convocatoria traerán una nueva dinámica en la publicación de las revistas en Colombia y por lo tanto ajustes en todos los procesos del ciclo de publicación en las revistas, esto implica que los resultados de los ajustes requeridos se verán a largo plazo (2-5 años) producto de los nuevos requerimientos del modelo de medición.

Cada revista según su modelo de gestión deberá implementar estrategias que permitan aumentar sus indicadores en los sistemas de medición más usados (JCR/SJR) y por lo tanto ser competitivas en el área de conocimiento respectivo.

Por lo anterior es importante reflexionar sobre el histórico de las publicaciones y como ellas deberán cambiar en un entorno internacional y cuánto tiempo tomarán los cambios para llegar a las más altas calificaciones.

Tomando como base las 28 revistas A1 de Publindex para el año 2016 y vigentes en el 2017, se pueden observar varios hechos significativos a partir de la revisión de la información de las revistas. La Tabla 1 presenta la información de las revistas A1 vigentes para el año 2017 (Publindex) tomada de Web of Science (WoS) y su base de datos SciELO Citation Index.

Es interesante observar algunos aspectos que serán elementos de discusión en la toma de decisiones en cuanto a las políticas de publicación de las revistas:

1) Al parecer existe una relación directa entre el h de la revista y el promedio de citas por documento, lo cual implicaría que la calidad (desde el punto de vista de citaciones por documento) es el factor determinante pero no exclusivo para obtener una revista de alta calidad e impacto.

2) Las revistas podrían tener un el número adecuado de artículos publicados en relación con el factor $\mathrm{h}$, esto implica que hay que analizar el comportamiento de las publicaciones en el área de conocimiento, no necesariamente a mayor número de publicaciones mayor $\mathrm{h}$.

3) Para las revistas A1 actuales, 16/28 revistas tienen un 50\% o más de autores de Colombia, esto implica que, aunque se cumpla con el porcentaje de autores externos a la entidad editora, las revistas colombianas se constituyen en la primera opción de publicación de autores colombianos. Se espera que el ingreso de la revista a WoS ó Scopus implique un cambio en el origen de los autores de las revistas colombianas. 
4) 26/28 de las revistas A1 tiene un $50 \%$ o más de artículos en español, aunque la mayoría de las revistas declaran varios lenguajes de publicación, la tendencia será la publicación al menos en dos idiomas inglés y español.

5) Las revistas deben revisar los diferentes tipos de documentos a publicar (de acuerdo con el área de conocimiento) puesto que el mayor porcentaje de documentos son artículos de investigación.

6) Normalmente los artículos de revisión son los más citados, pero se encuentra un bajo porcentaje de publicación de los mismos en la producción de documentos de las revistas.

7) 22/28 de las revistas A1 tienen la misma institución con el porcentaje mayor de artículos, esta relación cambiará a mediano debido a las políticas de autoría en el nuevo modelo de medición.

Por otra parte, Al revisar el JCR (Tabla 2) para las revistas colombianas se podría tener en cuenta:

i) La vida de citación de los documentos publicados (artículos, reviews, cartas al editor, comunicaciones cortas, etc) en su área de conocimiento.

ii) El área de clasificación de la revista implica tener más revistas pares con el mismo objetivo de subir de categoría. En las revistas colombianas, Biomédica compite con 19 revistas en su área mientras Caldasia compite con 211.

iii)Los tiempos de permanencia en los diferentes cuartiles, en general el tiempo de permanencia acumulado para las revistas colombianas es de 5 años como mínimo en la categoría Q4, esto implica que la gestión se debe hacer a largo plazo. Así pues, el futuro de las revistas en cada una de las instituciones en Colombia dependerá de una política institucional a largo plazo teniendo en cuenta la dinámica de la gestión del conocimiento a nivel local, regional, nacional e internacional para optimar los recursos y alcanzar el mejor impacto en la sociedad.

Tabla 1. Información de Wos (SciELO Citation Index) para las revistas A1 Publindex 2016-2017

\begin{tabular}{|c|c|c|c|c|c|c|c|c|c|}
\hline $\begin{array}{l}\text { Título de la } \\
\text { revista }\end{array}$ & $\begin{array}{l}\text { Entidad } \\
\text { Editora }\end{array}$ & $\begin{array}{l}\text { Docume } \\
\text { ntos } \\
\text { publica } \\
\text { dos }\end{array}$ & $\mathbf{h}$ & $\begin{array}{l}\text { Promedio } \\
\text { de citas } \\
\text { por } \\
\text { elemento }\end{array}$ & $\begin{array}{l}\text { Artículos } \\
\text { colombia } \\
\text { nos }(\%)\end{array}$ & $\begin{array}{l}\text { Artículos } \\
\text { publicados } \\
\text { en español } \\
(\%)\end{array}$ & $\begin{array}{l}\text { Artículos de } \\
\text { investigació } \\
\text { n (\%) }\end{array}$ & $\begin{array}{l}\text { Artícu } \\
\text { los de } \\
\text { revisió } \\
\text { n }(\%)\end{array}$ & $\begin{array}{l}\text { Institución } \\
\text { con mayor } \\
\text { número de } \\
\text { artículos } \\
(\%)\end{array}$ \\
\hline $\begin{array}{l}\text { Revista de } \\
\text { Salud Pública }\end{array}$ & $\begin{array}{l}\text { Universidad } \\
\text { Nacional de } \\
\text { Colombia }\end{array}$ & 980 & 14 & 2,32 & 56,74 & 82,55 & 91,53 & 1,33 & $\begin{array}{l}\text { Unal } \\
(26,53)\end{array}$ \\
\hline $\begin{array}{l}\text { UNIVERSIT } \\
\text { AS } \\
\text { PSYCHO- } \\
\text { LOGICA }\end{array}$ & $\begin{array}{l}\text { Pontificia } \\
\text { Universidad } \\
\text { Javeriana }\end{array}$ & 878 & 13 & 1,74 & 16,40 & 79,50 & 96,13 & 0,00 & PUJ $(11,73)$ \\
\hline BIOMÉDICA & $\begin{array}{l}\text { Instituto } \\
\text { Nacional de } \\
\text { Salud }\end{array}$ & 1042 & 13 & 1,82 & 80,33 & 89,44 & 63,82 & 5,57 & $\begin{array}{l}\text { UdeA } \\
(19,79)\end{array}$ \\
\hline $\begin{array}{l}\text { REVISTA } \\
\text { LATINOAM } \\
\text { ERICANA } \\
\text { DE }\end{array}$ & $\begin{array}{l}\text { Fundación } \\
\text { Universitari } \\
\text { a Konrad } \\
\text { Lorenz }\end{array}$ & 423 & 12 & 1,86 & 20,80 & 87,00 & 84,16 & 1,66 & $\begin{array}{l}\text { Universidad } \\
\text { de Granada } \\
(6,62)\end{array}$ \\
\hline
\end{tabular}


PSICOLOGÍ

A

\begin{tabular}{|c|c|c|c|c|c|c|c|c|c|}
\hline $\begin{array}{l}\text { COLOMBIA } \\
\text { MÉDICA }\end{array}$ & $\begin{array}{l}\text { Universidad } \\
\text { del Valle - } \\
\text { Univalle }\end{array}$ & 646 & 10 & 1,17 & 78,64 & 54,03 & 72,14 & 7,59 & $\begin{array}{l}\text { Univalle } \\
(38,7)\end{array}$ \\
\hline CALDASIA & $\begin{array}{l}\text { Universidad } \\
\text { Nacional de } \\
\text { Colombia }\end{array}$ & 347 & 9 & 1,54 & 80,40 & 76,66 & 99,71 & 0,00 & $\begin{array}{l}\text { Unal } \\
(53,03)\end{array}$ \\
\hline Aquichan & $\begin{array}{l}\text { Universidad } \\
\text { de la Sabana } \\
\text { - Unisabana }\end{array}$ & 362 & 9 & 1,75 & 38,12 & 78,45 & 87,57 & 4,14 & $\begin{array}{l}\text { Unal } \\
(12,43)\end{array}$ \\
\hline $\begin{array}{l}\text { ACTA } \\
\text { COLOMBIA } \\
\text { NA DE } \\
\text { PSICOLOGÍ } \\
\text { A }\end{array}$ & $\begin{array}{l}\text { Universidad } \\
\text { Católica de } \\
\text { Colombia }\end{array}$ & 334 & 9 & 1,44 & 41,07 & 82,44 & 86,31 & 7,43 & $\begin{array}{l}\text { Universidad } \\
\text { Catolica de } \\
\text { Colombia }\end{array}$ \\
\hline $\begin{array}{l}\text { REVISTA } \\
\text { COLOMBIA } \\
\text { NA DE } \\
\text { PSIQUIATRÍ } \\
\text { A }\end{array}$ & $\begin{array}{l}\text { Asociación } \\
\text { Colombiana } \\
\text { de } \\
\text { Psiquiatría }\end{array}$ & 793 & 9 & 0,91 & 79,45 & 94,96 & 75,16 & 4,92 & PUJ $(21,19)$ \\
\hline $\begin{array}{l}\text { REVISTA } \\
\text { COLOMBIA } \\
\text { NA DE } \\
\text { CIENCIAS } \\
\text { PECUARIAS }\end{array}$ & $\begin{array}{l}\text { Universidad } \\
\text { de } \\
\text { Antioquia - } \\
\text { Udea }\end{array}$ & 504 & 8 & 0,95 & 59,13 & 65,48 & 73,61 & 2,78 & $\begin{array}{l}\text { UdeA } \\
(34,52)\end{array}$ \\
\hline DYNA & $\begin{array}{l}\text { Universidad } \\
\text { Nacional de } \\
\text { Colombia }\end{array}$ & 1210 & 7 & 0,82 & 67,52 & 53,14 & 100,00 & 0,00 & $\operatorname{Unal}(36,53)$ \\
\hline $\begin{array}{l}\text { REVISTA } \\
\text { COLOMBIA } \\
\text { NA DE } \\
\text { ENTOMOLO } \\
\text { GÍA }\end{array}$ & $\begin{array}{l}\text { Sociedad } \\
\text { Colombiana } \\
\text { de } \\
\text { Entomologí } \\
\text { a }\end{array}$ & 590 & 7 & 1,07 & 50,17 & 73,05 & 92,20 & 3,56 & $\begin{array}{l}\text { Unal } \\
(15,93)\end{array}$ \\
\hline PROFILE & $\begin{array}{l}\text { Universidad } \\
\text { Nacional de } \\
\text { Colombia }\end{array}$ & 265 & 7 & 1,25 & 57,74 & 98,87 & 90,94 & 0,00 & $\begin{array}{l}\text { UdeA } \\
(8,30)\end{array}$ \\
\hline $\begin{array}{l}\text { REVISTA } \\
\text { DE } \\
\text { ESTUDIOS } \\
\text { SOCIALES }\end{array}$ & $\begin{array}{l}\text { Universidad } \\
\text { de los } \\
\text { Andes }\end{array}$ & 631 & 7 & 0,61 & 27,89 & 96,67 & 89,38 & 1,90 & $\begin{array}{l}\text { Uniandes } \\
(28,69)\end{array}$ \\
\hline $\begin{array}{l}\text { REVISTA } \\
\text { MVZ } \\
\text { CÓRDOBA }\end{array}$ & $\begin{array}{l}\text { Universidad } \\
\text { de Córdoba }\end{array}$ & 506 & 7 & 0,85 & 56,92 & 68,97 & 82,61 & 3,95 & $\begin{array}{l}\text { Unicor } \\
(20,36)\end{array}$ \\
\hline $\begin{array}{l}\text { AVANCES } \\
\text { EN } \\
\text { PSICOLOGÍ } \\
\text { A } \\
\text { LATINOAM } \\
\text { ERICANA }\end{array}$ & $\begin{array}{l}\text { Colegio } \\
\text { Mayor de } \\
\text { Nuestra } \\
\text { Señora del } \\
\text { Rosario }\end{array}$ & 267 & 6 & 0,89 & 34,08 & 69,29 & 95,88 & 0,00 & $\begin{array}{l}\text { Universidad } \\
\text { del Rosario } \\
(10,49)\end{array}$ \\
\hline
\end{tabular}




\begin{tabular}{|c|c|c|c|c|c|c|c|c|c|}
\hline VITAE & $\begin{array}{l}\text { Universidad } \\
\text { de } \\
\text { Antioquia }\end{array}$ & 259 & 6 & 1,07 & 86,10 & 73,75 & 87,26 & 2,70 & $\begin{array}{l}\text { UdeA } \\
(37,06)\end{array}$ \\
\hline INNOVAR & $\begin{array}{l}\text { Universidad } \\
\text { Nacional de } \\
\text { Colombia }\end{array}$ & 630 & 6 & 0,49 & 33,65 & 83,49 & 84,29 & 9,21 & $\begin{array}{l}\text { Unal } \\
(26,51)\end{array}$ \\
\hline $\begin{array}{l}\text { REVISTA } \\
\text { FACULTAD } \\
\text { DE } \\
\text { INGENIERÍ } \\
\text { A - } \\
\text { UNIVERSID } \\
\text { AD DE } \\
\text { ANTIOQUIA }\end{array}$ & $\begin{array}{l}\text { Universidad } \\
\text { de } \\
\text { Antioquia }\end{array}$ & 702 & 5 & 0,43 & 65,53 & 59,97 & 98,86 & 0,00 & $\begin{array}{l}\text { UdeA } \\
(23,22)\end{array}$ \\
\hline $\begin{array}{l}\text { Psicología } \\
\text { desde el } \\
\text { Caribe }\end{array}$ & $\begin{array}{l}\text { Universidad } \\
\text { del Norte }\end{array}$ & 187 & 5 & 0,71 & 62,03 & 95,72 & 90,91 & 0,00 & $\begin{array}{l}\text { Uninorte } \\
(20,86)\end{array}$ \\
\hline $\begin{array}{l}\text { HISTORIA } \\
\text { CRÍTICA }\end{array}$ & $\begin{array}{l}\text { Universidad } \\
\text { de los } \\
\text { Andes }\end{array}$ & 556 & 5 & 0,37 & 25,36 & 99,46 & 89,93 & 5,34 & $\begin{array}{l}\text { Uniandes } \\
(22,30)\end{array}$ \\
\hline $\begin{array}{l}\text { REVISTA } \\
\text { COLOMBIA } \\
\text { NA DE } \\
\text { ESTADÍSTI } \\
\text { CA }\end{array}$ & $\begin{array}{l}\text { Universidad } \\
\text { Nacional de } \\
\text { Colombia }\end{array}$ & 250 & 4 & 0,58 & 43,60 & 37,60 & 100,00 & 0,00 & Unal $(31,2)$ \\
\hline $\begin{array}{l}\text { CT\&F - } \\
\text { CIENCIA, } \\
\text { TECNOLOG } \\
\text { ÍA Y } \\
\text { FUTURO }\end{array}$ & $\begin{array}{l}\text { Instituto } \\
\text { Colombiana } \\
\text { de Petróleo }\end{array}$ & 188 & 4 & 0,32 & 94,15 & 7,98 & 97,34 & 0,00 & $\begin{array}{l}\text { ECOPETR } \\
\text { OL } \\
(32,45)\end{array}$ \\
\hline $\begin{array}{l}\text { INGENIERÍ } \\
\text { A E } \\
\text { INVESTIGA } \\
\text { CIÓN }\end{array}$ & $\begin{array}{l}\text { Universidad } \\
\text { Nacional de } \\
\text { Colombia }\end{array}$ & 652 & 4 & 0,53 & 72,24 & 59,20 & 94,48 & 2,61 & $\begin{array}{l}\text { Unal } \\
(45,09)\end{array}$ \\
\hline $\begin{array}{l}\text { Psychologia: } \\
\text { avances de la } \\
\text { disciplina }\end{array}$ & $\begin{array}{l}\text { Universidad } \\
\text { San } \\
\text { Buenaventur } \\
\text { a }\end{array}$ & 113 & 4 & 0,61 & 72,57 & 95,58 & 87,61 & 0,00 & $\begin{array}{l}\text { Universidad } \\
\text { San } \\
\text { Buenaventu } \\
\text { ra }(30,09)\end{array}$ \\
\hline $\begin{array}{l}\text { REVISTA } \\
\text { COLOMBIA } \\
\text { NA DE } \\
\text { PSICOLOGÍ } \\
\text { A }\end{array}$ & $\begin{array}{l}\text { Universidad } \\
\text { Nacional de } \\
\text { Colombia }\end{array}$ & 149 & 4 & 0,53 & 42,28 & 78,52 & 84,56 & 0,00 & $\begin{array}{l}\text { Unal } \\
(20,81)\end{array}$ \\
\hline $\begin{array}{l}\text { PENSAMIE } \\
\text { NTO } \\
\text { PSICOLÓGI } \\
\text { CO }\end{array}$ & $\begin{array}{l}\text { Pontificia } \\
\text { Universidad } \\
\text { Javeriana - } \\
\text { PUJ - Sede } \\
\text { Cali }\end{array}$ & 130 & 3 & 0,38 & 35,39 & 95,39 & 95,39 & 0,00 & PUJ $(6,16)$ \\
\hline $\begin{array}{l}\text { CUADERNO } \\
\text { S DEL } \\
\text { DESARROL } \\
\text { LO RURAL }\end{array}$ & $\begin{array}{l}\text { Pontificia } \\
\text { Universidad } \\
\text { Javeriana }\end{array}$ & 139 & 3 & 0,53 & 19,42 & 81,30 & 94,96 & 0,00 & $\operatorname{PUJ}(7,12)$ \\
\hline
\end{tabular}


Tabla 2. Ranking de Revistas colombianas en JCR

\begin{tabular}{|c|c|c|c|c|c|c|}
\hline \multicolumn{4}{|c|}{ InCites ${ }^{\text {"' J Journal Citation Reports }}{ }^{\circ}$} & \multicolumn{3}{|c|}{ THOMSON REUTERS } \\
\hline Ranking & Título de la revista & $\begin{array}{l}\text { Citas } \\
\text { totales }\end{array}$ & $\begin{array}{c}\text { Factor de } \\
\text { impacto de } \\
\text { la revista } \\
\end{array}$ & $\begin{array}{l}\text { Número de años } \\
\text { en categoría Q4 }\end{array}$ & $\begin{array}{c}\text { Número de } \\
\text { años en } \\
\text { categoría Q3 }\end{array}$ & $\begin{array}{c}\text { Ranking } \\
2016\end{array}$ \\
\hline 1 & Colombia Médica & 259 & 0,733 & $2010-2014(5)$ & $2015-2016(2)$ & $112 / 154$ \\
\hline 2 & Biomédica & 759 & 0,727 & $2009-2016(8)$ & & $16 / 19$ \\
\hline 3 & $\begin{array}{c}\text { REVISTA } \\
\text { LATINOAMERICANA } \\
\text { DE PSICOLOGÍA }\end{array}$ & 299 & 0,717 & $\begin{array}{c}1997-2001, \\
2004- \\
2005,20072009- \\
2010,2012-2015 \\
(11)\end{array}$ & $\begin{array}{c}2016,2011 \\
2008,2006(3)\end{array}$ & $92 / 128$ \\
\hline 4 & $\begin{array}{l}\text { Earth Sciences } \\
\text { Research Journal }\end{array}$ & 102 & 0,634 & 2009-2016 (8) & & $176 / 188$ \\
\hline 5 & $\begin{array}{c}\text { Cuadernos de } \\
\text { Desarrollo Rural }\end{array}$ & 87 & 0,621 & $2010-2015(6)$ & $2016(1)$ & $34 / 56$ \\
\hline 6 & $\begin{array}{l}\text { Universitas } \\
\text { Psychologica }\end{array}$ & 376 & 0,313 & $\begin{array}{c}2011,2013-2016 \\
\text { (5) }\end{array}$ & $2012(1)$ & $122 / 128$ \\
\hline 7 & $\begin{array}{c}\text { CT\&F-Ciencia } \\
\text { Tecnologia y Futuro }\end{array}$ & 76 & 0,310 & $2010-2016(6)$ & & $87 / 92$ \\
\hline 8 & Revista MVZ Cordoba & 148 & 0,286 & 2009-2016 (8) & & $51 / 58$ \\
\hline 9 & $\begin{array}{l}\text { Ingeniería e } \\
\text { Investigación }\end{array}$ & 108 & 0,280 & $2012-2016(5)$ & & $83 / 85$ \\
\hline 10 & $\begin{array}{l}\text { Revista de Estudios } \\
\text { Sociales }\end{array}$ & 143 & 0,267 & $2011-2016(6)$ & & $35 / 41$ \\
\hline 11 & $\begin{array}{c}\text { REVISTA } \\
\text { COLOMBIANA DE } \\
\text { ENTOMOLOGIA }\end{array}$ & 271 & 0,253 & 2007-2016 (10) & & $88 / 91$ \\
\hline 12 & $\begin{array}{l}\text { Revista Colombiana de } \\
\text { Ciencias Pecuarias }\end{array}$ & 134 & 0,242 & $2011-2016(6)$ & $2010(1)$ & $53 / 58$ \\
\hline 13 & CALDASIA & 256 & 0,241 & $2010-2016(5)$ & & $206 / 211$ \\
\hline 14 & $\begin{array}{l}\text { Academia-Revista } \\
\text { Latinoamericana de } \\
\text { Administración }\end{array}$ & 64 & 0,237 & $2009-2016(8)$ & & $117 / 121$ \\
\hline 15 & Historia Crítica & 75 & 0,175 & $\begin{array}{l}\text { 2009-2010, } \\
2012-2016(7)\end{array}$ & 2011 (1) & $74 / 87$ \\
\hline
\end{tabular}

Fiderman Machuca-Martínez

Editor-Jefe

Revista Ingeniería y Competitividad

Universidad del Valle

Cali, Colombia 